\title{
Enfoque organizacional de la selección del personal y las competencias laborales desde la
} Economía Política

\section{Organizational approach in the selection of personnel and labor capability from Political Economy}

Juan Carlos Castillo Moya. ${ }^{1}$, Marianela Bermejo Salmon. ${ }^{2}$, Raquel Ivon Flores León. ${ }^{3}$ \& Esteban Mauricio Castillo Noboa. ${ }^{4}$

\section{DOI: https://doi.org/10.33262/visionariodigital.v3i3.754}

\begin{abstract}
It was analyzed the contradiction between the process of selection of Human Resources and labor competition from an economic and political point of view in the "Escuela Superior Politécnica de Chimborazo". Utilizing the combination of methods and techniques as: logic-historic, synthesis Analysis, induction, deduction, experts point of views and others. As a principal result of the study a presentation and analysis was made of the existing contradictions between the process before mentioned in the three fundamentals moments: the actual behavior, the cause and
\end{abstract}

\footnotetext{
${ }^{1}$ Magister en Seguridad Industrial mención Prevención de Riesgos y Salud Ocupacional, Docente de la Escuela Superior Politécnica de Chimborazo, Ecuador, jrjdcastillo@gmail.com

2 Doctora en Ciencias Económicas, Facultad de Ciencias Económicas y Empresariales, Universidad de Oriente, Ecuador, marianela@uo.edu.cu

${ }^{3}$ Psicóloga Educativa, Gobierno Autonomo Descentralizado Municipal Riobamba, Ecuador, flrakelivon.25@gmail.com

${ }^{4}$ Licenciado en Ciencias de la Educación mención Físico Matemático, Unidad Educativa la Salle, Ecuador, estebancastillo@riobambalasalle.edu.ec
} 
the effects of which derived and from that function and methods of study of Political Economy, were able to be treated in a way that it was possible the selection process of Human Resources and competitions in the context of Organizational labor.

Keywords: Selection process, labor capability, human resources, cognitive function, methodological function, ideological function.

\section{RESUMEN}

Se analizaron las contradicciones del proceso de selección del talento humano y las competencias laborales desde un enfoque económico-político en la Escuela Superior Politécnica de Chimborazo. Se utilizó la combinación de métodos y técnicas tales como: histórico-lógico, análisis y síntesis, inducción- deducción, criterios de expertos, entre otros. Como resultados principales del estudio se realizó la presentación y análisis de las contradicciones existentes en los procesos antes citados en tres de sus momentos fundamentales: su comportamiento actual, las causas que las suscitaron y los efectos que de ellas se derivaron, y cómo a partir del objeto, funciones y método de estudio de la economía política, pudieron ser tratados de forma tal que posibilitaron la realización del proceso de selección del talento humano y sus competencias laborales en el contexto organizacional.

Palabras clave: Proceso de selección, competencias laborales, talento humano, función cognoscitiva, función metodológica, función ideológica.

\section{INTRODUCCIÓN}

El ser humano en su doble condición de ser biológico y de ser social es portador de la fuerza de trabajo, siendo un elemento decisivo de las fuerzas productivas y sujeto de las relaciones de producción.

El hombre tiene entre otras capacidades, las de crear, producir y generar; lo que le convierte en el elemento más importante de la empresa porque es él, el que hará de la empresa creativa, productiva y generadora, no solo desde una perspectiva económica sino, también 
más humana, solidaria y responsable del mejoramiento de la calidad de vida de la comunidad y del país.

Se exige que el ser humano desarrolle al máximo su talento y creatividad para enfrentar el cambio y la innovación, desde las investigaciones científicas en los centros de educación superior.

La creación de condiciones que permitan en las universidades, una adecuada gestión del talento humano, constituye una condición necesaria para alcanzar el umbral de excelencia requerido por la Ley Orgánica de Educación Superior del Ecuador y esto a su vez implica una gestión holística de los procesos de contratación del personal y competencias laborales que garanticen niveles superiores del desempeño del talento humano.

Tal es el caso de la Escuela Superior Politécnica de Chimborazo, ubicada en la provincia de Chimborazo y específicamente en la ciudad de Riobamba, la cual se ha constituido en una de las más emblemáticas instituciones de Educación Superior en Ecuador. En la actualidad es una de las mejores universidades públicas del país, no obstante, existen una serie de dificultades a partir del tratamiento actual del proceso de selección y competencias laborales del personal docente que pueden limitar su condición de vanguardia. De ahí, la necesidad de realizar investigaciones con un rigor científico, que permitan ante todo conocer las causas, manifestaciones y posibles soluciones que puedan sugerirse en el período 2017 y 2018 en lo cual es importante considerar a la economía política.

Teniendo en cuenta lo antes planteado este trabajo tiene como problema científico: ¿Cómo incide en el proceso de selección del talento humano y las competencias laborales la inexistencia desde un enfoque económico-político de sus contradicciones en la Escuela Superior Politécnica de Chimborazo (ESPOCH) del Ecuador?

Hipótesis: Si las contradicciones existentes en el proceso de selección del talento humano y las competencias laborales son asimiladas desde un enfoque económico-político posibilitarían su adecuado tratamiento y a su vez sugerir soluciones para el perfeccionamiento de este proceso en la Escuela Superior Politécnica de Chimborazo. Por lo que el objetivo de este estudio está dado en el análisis de las contradicciones 
existentes en el proceso de selección del talento humano y las competencias laborales desde un enfoque económico-político en la Escuela Superior Politécnica de Chimborazo. Los resultados principales se enmarcan en dos partes; inicialmente se hace una valoración general de la economía política como ciencia, la segunda parte se centra en la fundamentación de las contradicciones existentes en los procesos antes citados, en tres de sus momentos fundamentales: su comportamiento actual, las causas que los suscitan y los efectos que de ellas se derivan.

\section{MÉTODOS UTILIZADOS}

Durante la realización de la investigación se empleó desde el enfoque de la Economía Política el método del materialismo dialéctico e histórico, en su acepción más precisa con la concepción materialista de la historia que comprende los métodos siguientes:

- Histórico - Lógico: fue empleado para la descripción de la evolución de los procesos de competencias laborales y organización del trabajo.

- Análisis y Síntesis: se utilizó fundamentalmente en la primera fase del trabajo, en el procesamiento de la bibliografía consultada y su análisis crítico.

- Métodos empíricos: fueron aplicadas las técnicas utilizadas en el desarrollo de la investigación, tales como: entrevistas, cuestionarios de opinión, trabajo grupal, observación directa entre otras.

\section{RESULTADOS Y DISCUSIÓN}

En el sentido más amplio de la palabra, la economía política, es la ciencia que estudia las leyes que rigen la producción y el intercambio de los medios materiales de vida en la sociedad humana",.

En el sentido más estrecho, la economía política, es la ciencia que estudia un material histórico concreto: el conjunto de relaciones sociales que se establecen en un proceso de producción específico.

¿Qué son las relaciones sociales de producción?

${ }^{5}$ Engels F: “Anti During, página 179. 
Las relaciones sociales de producción son el conjunto de relaciones económicas que se establecen entre los hombres, independientemente de su conciencia y de su voluntad, en el proceso de producción, distribución, cambio y consumo. Existe una estrecha relación e interdependencia entre estas fases. La producción determina la distribución, el cambio y el consumo, pero estas no juegan un papel pasivo, sino que influyen decisivamente en el proceso de producción.

La producción social solo puede darse cuando los hombres se unen para obrar en común, para establecer un intercambio de actividades, y es esto precisamente la base de la existencia de la humanidad, de ahí su importancia.

La base de las relaciones sociales de producción se encuentra en las relaciones de propiedad sobre los medios de producción. La propiedad como categoría económica expresa las relaciones que se establecen entre los hombres en la apropiación de las premisas y resultados de la producción. El carácter de las relaciones de producción depende de quiénes sean los dueños de los medios de producción, de cómo se realice la unión de esos medios con los productores.

Está demostrado que en la producción social de su vida los hombres establecen determinadas relaciones necesarias e independientes de su voluntad, relaciones de producción que corresponden a una fase determinada de desarrollo de sus fuerzas productivas.

A su vez las fuerzas productivas son el conjunto de los medios de producción y de los hombres que los emplean para producir bienes materiales. Los elementos materiales de las fuerzas productivas que funcionan en la producción social bajo determinadas formas económicas constituyen la base material y técnica de la sociedad.

En la época actual, se convierte en fuerza directamente productiva la ciencia. La fuerza productiva principal está formada por los trabajadores, que crean los instrumentos de producción, los ponen en movimiento, poseen experiencia y hábitos de trabajo.

Las fuerzas productivas expresan la relación que existe entre el hombre y los objetos y fuerzas de la naturaleza, el grado en que este los domina 
Lo que distingue a la historia humana de la historia del resto de la naturaleza, incluyendo a los otros animales, es que el hombre a través del tiempo ha aprendido a producir los medios de su propia existencia, por lo tanto, los hombres producen su propia vida material. Esta producción provoca que pueda actuar sobre la naturaleza, y es por medio de esta práctica que modifica el mundo social y natural. De ahí que, el hecho de que los seres humanos aprendan a ser más productivos, a crear más y mejores objetos, hace posible las nuevas formas de vivir donde; el conocimiento científico se logra en la práctica de la transformación de la naturaleza y esta se convierte en agente de la transformación de la naturaleza humana, su evolución va a la par con los cambios en el modo de producción.

En estos comportamientos históricos, las relaciones de producción han ido cambiando y transformándose, de acuerdo al ritmo que han impuesto la tecnología, lo que incide directamente en la participación del trabajo humano en los procesos y actividades de transformación, de ahí la importancia de analizar y determinar la participación que ha tenido $\mathrm{y}$ tienen las fuerzas productivas, específicamente el factor humano, recursos humanos, capital humano, talento humano u otras terminologías que suelen utilizarse indistintamente en tal sentido de cualquier institución, mucho más de las que prestan y brindan servicios, como es la educación tal es el caso específico de Ecuador donde el talento humano es una de las acepciones más utilizadas.

Hoy en día el mundo se ha convertido en una sociedad compuesta por Politécnicas y Universidades, las cuales están formadas por recursos tanto tecnológicos, financieros, físicos como humanos, sin embargo, estos últimos tienen un rol protagónico, debido a que el éxito o fracaso de cualquier institución de Educación Superior, así como de quienes la dirigen, está vinculado con la calidad de personas que prestan sus servicios en las diferentes áreas. Como señala Bok, D. (1992) "La calidad de las instituciones de educación superior se mide por la calidad de sus estudiantes y de sus profesores".

Lo anteriormente expuesto permite intuir que para satisfacer las exigencias de la sociedad se debe estar preparado para garantizar la calidad requerida en el contexto organizacional desde su enfoque de gestión sistémica que teniendo en cuenta en su accionar la gestión por competencias y la inserción de las competencias laborales, por procesos y organizacionales, 
consolide la prestación de los servicios de la Educación Superior vinculados al desarrollo de un país y su entorno; donde medir el desempeño del personal resulta imprescindible a partir de realizar una correcta selección tanto desde lo conceptual, metodológico y práctico, en lo cual la exigencia de la evaluación y certificación de las competencias demandadas, adquiridas, desarrolladas y consolidadas constituyen un mecanismo de control y validación en muchas profesiones, para garantizar a los usuarios la autenticidad y la capacidad profesional.

Lo antes citado guarda una estrecha correspondencia con las necesidades y expectativas de diferentes instituciones de educación superior de Ecuador, y en tal sentido el Concejo de Evaluación y Acreditación de la Calidad de Educación Superior (CEACES) que es una institución que se encarga de reconocer a las universidades y politécnicas el nivel o grado de categoría; le confirió a la Escuela Superior Politécnica de Chimborazo (ESPOCH) la categoría A atendiendo a la calidad de profesionales, tecnología de apoyo, potencialidad de sus egresados, vinculación de estos con la colectividad y las necesidades y expectativas que al respecto enfrentan y promueven diferentes acciones estratégicas para brindar solución a las situaciones que se presenten en este contexto; de todo ello se identificaron deficiencias en concebir los factores antes expuestos de manera sistémica desde un enfoque de gestión, fundamentado en el estatuto politécnico vigente, y en las reformas ecuatorianas; así como también con las nuevas tareas pedagógicas y los requerimientos de ser eficientes en su gestión y obtener resultados satisfactorios en los procesos de evaluación, para lo cual considerar el rol que en ello puede desempeñar la implicación de la economía política, desde el cumplimiento de sus funciones, tiene extraordinaria utilidad para las investigaciones científicas en especial para las ciencias económicas.

La economía política cumple cuatro funciones básicas: cognoscitiva, metodológica, práctica e ideológica.

La función cognoscitiva, orienta hacia el estudio de las relaciones sociales de producción que se establecen entre los hombres en el proceso de producción, unida orgánicamente con las fuerzas productivas. Representa el sistema de conocimientos que sustenta la teoría de dicha ciencia. 
La función metodológica, está dirigida a servir de fundamento teórico del resto de las ciencias económicas, tanto de aquellas que explican los aspectos funcionales y sectoriales de la sociedad, como de ciencias económicas que se relacionan con otras ramas del conocimiento.

Al estudiar la economía política el sistema de las relaciones sociales de producción proporciona: la teoría general que sirve de base para las diversas ciencias sociales y el método que permite investigar la esencia, contradicciones y leyes que regulan los procesos económicos cuyas formas externas, es decir al nivel de la manifestación concreta, investigan las demás ciencias económicas: contabilidad, las finanzas, administración de empresa, entre otras.

El método de la Economía Política Marxista Leninista es el materialismo dialectico. Este método parte de que en la naturaleza y en la sociedad, todos los fenómenos y relaciones no existen para siempre, eternos e inmutables, sino que se encuentran en proceso de desarrollo y transformación. Implica la investigación de todo el conjunto de hechos, para poner al descubierto la esencia que ocultan; analizar, ordenar y sintetizar los datos históricos, las teorías y enfoques que le preceden y las experiencias prácticas, lo puede hacerse subiendo el peldaño del pensamiento científico a las abstracciones.

Tanto la función cognoscitiva como la metodológica no pueden ser analizadas sin considerar la función práctica. Relacionado con la función práctica de la Economía Política debe destacarse que su objetivo, al igual que el de cualquier ciencia, no es sólo describir y explicar el mundo, sino también transformarlo en función de los intereses de las grandes masas ${ }^{6}$. Y es esto lo que explica que la Economía Política fundamente científicamente la política económica del Estado. En el caso específico de esta investigación se expresa en la propuesta de posibles soluciones a las contradicciones que se han suscitado durante el acontecer del proceso de selección y competencias laborales en la ESPOCH de Ecuador.

${ }^{6} \mathrm{C}$. Marx: Tesis sobre Feuerbach (1845). 
La función ideológica se relaciona con el carácter clasista de la ciencia que significa que sus descubrimientos y conclusiones siempre responden a los intereses y a la práctica social de una clase determinada. En el caso de la Economía Política Marxista Leninista, la misma demuestra científicamente la necesidad de la lucha de clases, el papel histórico del proletariado en la lucha contra el capitalismo y la necesidad de la construcción del Socialismo.

Las funciones de la Economía Política deben ser analizadas en su interrelación mutua, donde conocimientos, teoría, ideología y realidad práctica, muestran no solo los problemas derivados de las relaciones de producción sino también posibles soluciones a las contradicciones que estas expresan.

El tener en cuenta estas funciones garantiza: contextualizar la investigación, a través del análisis de los fundamentos teóricos y metodológicos que permiten el tratamiento de las contradicciones inherentes al proceso de selección sobre la base de las competencias laborales en la ESPOCH.

\section{Contradicciones suscitadas en el proceso de selección}

Muchas instituciones, desde sus Áreas del Talento Humano, intentan alcanzar la excelencia y se esfuerzan para que día a día los trabajadores gocen de un ambiente laboral acogedor y proclive a la eficiencia, no obstante, es común encontrar ciertos problemas que se repiten en la mayoría de estas, independientemente del sector al que pertenezcan. Son situaciones que a veces parecen simples pero que pueden acarrear graves consecuencias en el desenvolvimiento de la institución. A continuación, se relacionan los clásicos problemas que se presentan en la Gestión de los Recursos Humanos por competencias:

- Falta de capacitación: Cuando una persona que forma parte de un equipo y que ocupa un puesto con unas funciones determinadas no ha recibido la capacitación que requiere su trabajo, se reducen notablemente las posibilidades de que sus tareas sean desarrolladas de manera eficiente.

- Falta de concienciación: El segundo gran problema al que se enfrenta normalmente un departamento de Recursos Humanos es la falta de concienciación. Esto se produce 
cuando uno de los miembros del equipo no quiere hacer su trabajo de la manera correcta, afectando a la empresa y a sí mismo.

- Los líderes a veces no se dan cuenta de la gran pérdida que esto supone ya que para que exista la eficiencia es absolutamente necesario que tanto la empresa como el empleado tengan un acuerdo beneficioso por ambas partes para que, gracias a la satisfacción mutua, se pueda trabajar de la mejor manera posible.

- Métodos de selección poco apropiados: Los encargados del reclutamiento y selección deben tener habilidades para detectar a las personas adecuadas para cada posición, limitando así el riesgo de equivocarse, algo que, aunque siempre existe, se debe intentar minimizar. Buscar un encuentro entre lo que se necesita realmente en la empresa y lo que la persona candidata puede ofrecer, es considerado casi un arte para el que hay que aprender a detectar perfiles y conocer a fondo las tareas de cada puesto.

- Exceso o falta de personal: Otro de los problemas más frecuentes es el exceso o falta de personal. Cuando el personal es excesivo, casi siempre se puede hablar también de "burocracia excesiva". En estos ambientes es posible que los procesos sean muy complejos y poco claros y los costes se elevan de tal manera que se inicia el declive. Lo opuesto a esta situación es la falta de personal. En las empresas con pocas personas, los trabajadores suelen tener disímiles usos, algo que, aunque a corto plazo puede ser un ahorro, a largo plazo se convierte en una gran pérdida de talentos y de eficiencia.

- Problemas de comunicación: Los problemas de comunicación son fallos que se pueden dar en el ámbito de lo interpersonal o a partir de los jefes hacia los empleados. La consecuencia común a todos ellos es el mal clima laboral, un espacio determinado por el estilo agresivo en la comunicación. Los conflictos y rivalidades con las que ha de lidiar el departamento de Recursos Humanos conducen a problemas de coordinación y a falta de solidaridad en el día a día.

- Inobservancia de las legislaciones y normativas jurídicas y técnicas que rigen la actividad: Muchos especialistas y técnicos no cumplen lo establecido legalmente 
cometiendo violaciones y como resultado calificaciones deficientes o malas en las auditorias o inspecciones externas.

- Evaluaciones del desempeño a los trabajadores formales: En la mayoría de las empresas se realizan las evaluaciones del desempeño del trabajador sin tener en cuenta las competencias y los resultados concretos según las funciones y actividades del puesto.

- La falta de previsión, la inflexibilidad y la actitud defensiva suelen ser otros de los problemas graves que muy comúnmente se encuentran en Recursos Humanos: Cuando una empresa acepta y entiende que lo mejor que tiene es su personal, empieza a trabajar en pos de conseguir que sea ese recurso la base de su éxito. Solo de esta manera se puede empezar a pensar en la excelencia.

Las Instituciones de Educación Superior (IES) de Ecuador aunque no en su generalidad, no escapan a estas transformaciones en el orden social, económico, cultural entre otros y en tal sentido un gran número de estas transitan aún por el Plan del Buen Vivir en el que no obstante a sus aciertos; al no estar contemplado en este como un proceso único la selección del personal desde un enfoque de competencias ha ocasionado algunos desaciertos aunque estas desde sus departamentos de recursos humanos, intentan alcanzar la excelencia y se esfuerzan para que día a día los trabajadores estén imbuidos en un clima laboral grato y propenso a la eficiencia, no obstante, es común encontrar ciertos problemas que se repiten en todas ellas, independientemente del ámbito en que se insertan.

Resultan situaciones aparentemente no complejas pero que pueden conducir a notables efectos desde su impacto económico, político y social s en el desenvolvimiento de la institución y que se resumen en el contexto en que se desarrolla este estudio. En consonancia con las situaciones antes expuestas, seguidamente los autores expresan sus juicios de valor respecto a algunas de las contradicciones identificadas en el transcurso de este estudio, sus causas y efectos.

\section{Contradicciones de carácter:}

Cultural. Existencia de una cultura organizacional que no prioriza el tratamiento sistémico del proceso de selección sobre la base de las competencias laborales.

\section{Causas de esta contradicción:}


- Falta de identidad del rol que desempeña dentro del sistema institucional, la selección del personal y las competencias laborales.

- La no cuantificación y cualificación del nivel de preparación de los recursos humanos y la insuficiente gestión del mismo en los procesos analizados.

\section{Efectos:}

- Insuficiente claridad de qué se espera de cada trabajador, es decir: qué competencias y qué resultados dentro del proceso de selección e integración.

- Se obvian las diferencias individuales de cada trabajador representada en lo fundamental por sus competencias.

- No existe la necesaria socialización del rol que desempeñan en este proceso; las funciones cognoscitivas, metodológicas, ideológicas y prácticas, se niega, además; la dialéctica de los procesos analizados y su impacto social.

Contradicciones de carácter de Centralización y/o subjetividad: Existe una incoherencia entre la cultura organizacional y la expresión de las competencias que esta debe promover, en los procesos de reclutamiento - selección del personal y su repercusión en la evaluación del desempeño.

Causas de esta contradicción: Los mecanismos utilizados para el proceso de reclutamiento y selección no se centran en políticas y acciones que propendan la ejecución de este proceso alineado a las competencias laborales, en un mercado cada vez más competitivo.

\section{Efectos:}

- Inexistencia de un proceso de retroalimentación continúo y compartido de la información requerida en la gestión del proceso de reclutamiento y selección del personal.

- Existe una alta centralización y subjetividad en el proceso de reclutamiento y selección del personal que no posibilita la participación democrática de los trabajadores, negándose el carácter dialéctico del impacto económico y social de este proceso. 
Contradicciones de carácter Económico: Se manifiestan en el incumplimiento de los indicadores directivos y límites establecidos para las Instituciones de Educación Superior (IES) por el Ministerio de Finanzas como resultado de las incongruencias entre los rendimientos obtenidos por el personal seleccionado y sus competencias.

\section{Causas de esta contradicción:}

- No correspondencia entre los resultados del trabajo y las competencias laborales para el cumplimiento de las funciones atribuibles a cada puesto de trabajo.

- Inadecuada aplicación de la política salarial que no considera en su núcleo las diferencias individuales del personal en función de sus competencias.

- Aplicación de una conducta económica que no propicia los niveles de eficiencia y eficacia planificados.

\section{Efectos}

- Incumplimiento de los servicios ofertados desde lo cualitativo y cuantitativo se disminuye la cantidad de servicios que se ofertan esencialmente desde las asesorías metodológicas e investigativas y la disminución de afluencia al mercado de trabajo que esta IES oferta.

- Salarios que no se corresponden con los resultados individuales de cada trabajador.

- Decrecimiento de la eficiencia, la eficacia económica y la productividad del trabajo.

- Se soslayan las funciones fundamentalmente en lo cognitivo, metodológico y práctico, no se precisa el rol que desempeña cada trabajador dentro del proceso analizado y la apropiación de los resultados de su trabajo y su repercusión en la satisfacción de las necesidades que en este orden la sociedad ecuatoriana demanda.

Contradicciones de carácter de Dirección administrativa: Existencia de directivos que no propician que el personal cumplimente la misión y objetivos de la organización.

\section{Causas de esta contradicción:}

- Existencia de algunos directivos que no tienen un sentido creativo en su política de dirección y manifiestan una conducta que no resulta ser ni empática ni asertiva en el desempeño de sus funciones. 
- Estilos y métodos burocráticos de trabajo.

- Inestabilidad de los directivos en los puestos clave de dirección.

\section{Efectos:}

- Los trabajadores no se sienten comprometidos con la totalidad de sus directivos en la búsqueda de soluciones a los problemas existentes

- . Estancamiento de la iniciativa y motivación por el trabajo.

- Desmotivación e insatisfacción de los trabajadores

- Se evidencia, por parte de directivos, la percepción equívoca de no reconocer el carácter social de la propiedad sobre los medios de producción en la construcción de la sociedad.

Contradicciones de carácter Epistemológico: El proceso de selección no es considerado desde su acepción holística en un contexto histórico social bien definido y por tanto se soslayan los problemas que en algunos contextos se suscitan producto de la vertiginosidad y complejidad de un mundo cambiante en la que se va acentuando la tendencia globalizadora, que generan nuevos escenarios y nuevas variables como la competitividad, la calidad y la excelencia.

\section{Causas de esta contradicción:}

- No se percibe el proceso de selección en función de las competencias de las personas en su interacción con los puestos de trabajo, el ambiente laboral y la cultura organizacional.

- No se aplican los presupuestos teóricos y metodológicos existentes que permiten a través de la experiencia alcanzada un adecuado tratamiento del proceso objeto de análisis.

- Los procedimientos aplicados en el estudio y análisis de este proceso no representan el nexo de las conductas o desempeños individuales con la estrategia de la organización y el sistema de trabajo asumido.

\section{Efectos:}


- El proceso de selección y sus competencias no son asumidas desde el punto de su función e integralidad a través de presupuestos teóricos y metodológicos que, posibiliten su inserción en los procesos de gestión de los recursos humanos.

- No se logra el desempeño exitoso de los recursos humanos y, en especial, de los directivos, desde un alcance holístico.

- Predomina la gestión por métodos tradicionales que restan calidad a los resultados

obtenidos en el proceso de selección e integración del personal y que son portadores de quebrantado impacto social

Retos y desafíos que los autores de este estudio proponen para contrarrestar desde la concepción de la Economía Política las contradicciones antes expuestas y su impacto económico y social:

- Realizar un trabajo orientado a lograr la exigencia, control y mejora continua que caracterizan la naturaleza holística del proceso de selección del personal.

- Capacitar y formar a los cuadros, directivos, así como especialistas y técnicos encargados de gestionar el talento humano en las IES acorde a las políticas, legislaciones y normativas vigentes para esta actividad e instrumentar mecanismos para evaluar las acciones de aprendizaje.

- Propiciar y promocionar un ambiente innovador que posibilite el intercambio de conocimientos en torno a la gestión del talento humano enmarcado en la gestión por competencias como componente fundamental de las estrategias y políticas a desarrollar por las IES.

\section{Conclusiones.}

El desarrollo del trabajo realizado permite llegar a las consideraciones siguientes:

- El estudio y análisis del proceso de selección y las competencias laborales desde la Economía Política fortalece el tratamiento de las contradicciones suscitadas desde su esclarecimiento, comprensión y proyección de posibles soluciones que a su vez sientan las bases para el desarrollo de las fuerzas productivas y de las relaciones de producción en el Ecuador, en el contexto organizacional y su fortalecimiento como ser social. 
- El estudio de las funciones de la Economía Política y su consecuente aplicación contribuyó a despuntar la necesaria interrelación entre el proceso de selección del personal y sus competencias laborales lo que hará viable contar con un talento humano competente que a su vez contribuya al desarrollo, y consolidación del proceso de enseñanza-aprendizaje.

- El análisis del proceso de selección concebido desde el conocimiento de la Economía Política, ejerce un impacto positivo en lo fundamental en el orden teórico, metodológico, económico y social en el contexto analizado.

- La distribución justa de las riquezas creadas en las IES sobre la base del aporte de cada trabajador desde su puesto de trabajo, proceso y organización sin duda alguna contribuye a fortalecer la motivación de estos por los resultados de su trabajo y su sostenibilidad lo que redundará en el desarrollo económico y social de la sociedad ecuatoriana.

\section{Referencias Bibliografía}

- Anzil, Federico. "El progreso tecnológico y el crecimiento económico". Artículo disponible en el sitio web www.econlink.com.ar, febrero de 2009

- Cuesta Santos, A (2010). Tecnología de Gestión de Recursos Humanos. Tercera edición corregida y ampliada. La Habana. Editorial "Félix Varela y ampliada.

- Chiavenato, I. (2012). Administración de Recursos Humanos. México. Editorial Mc Graw Hill.

- Colectivo de autores (2004). Manual Fundamentos Generales del Modo de Producción Capitalista. La Habana. Editorial Félix Varela.

- Díaz Gispert, L (2007). La Gestión de los Recursos Humanos y su evolución con el desarrollo de la administración en Cuba. Cuba.

- ENGELS F: “Anti During. Editorial Progreso, 1989.

- Escandell Sosa, V (2019). Ciclo de Conferencias de Economía Política.

- Guevara, E (2006). Apuntes críticos a la Economía Política. La Habana. Editora de Ciencias Sociales.

- Lorenzo García, R. (2005). 'Predictores del talento'. Intangible Capital. Vol. 1. No.7, Barcelona España. 
Vol. 3, N³., p.281 - 280, julio - septiembre, 2019

- Lorenzo García, R. (2005). 'Competencias asociadas con el talento para la Dirección'. Gerencia en salud. Revista del Asociación Panamericana de Gestión de la Salud. Perú.

- Marx, C (1989). Contribución a la Crítica de la Economía Política. Editorial Progreso.

- Marx, C (1980). El Capital. Postfacio a la Segunda Edición, La Habana. Editorial Ciencias Sociales.

- Marx, C (1980). El Capital. Prefacio a la Primera Edición. La Habana. Editorial Ciencias Sociales.

- Marx, C, Engels, F. Manifiesto del Partido Comunista. Obras Escogidas en II Tomos. Tomo I. Moscú. Ediciones en Lenguas Extranjeras.

- Morales Pérez, M (2012). Curso de Economía Política Marxista Leninista. Rumiantsev, A.: Economía Política. Editorial Progreso, 1978.

- Pavón González, R (2006). Apuntes sobre las teorías de las competencias.

- Pacheco, U (2015). La economía política y la investigación científica. 


\section{PARA CITAR EL ARTÍCULO INDEXADO.}

Castillo Moya, J., Bermejo Salmon, M., Flores León, R., \& Castillo Noboa, E. M. (2019). Enfoque organizacional de la selección del personal y las competencias laborales desde la Economía Política. Visionario Digital, 3(3), 281298. https://doi.org/10.33262/visionariodigital.v3i3.754

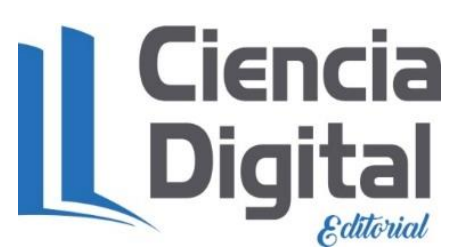

El artículo que se publica es de exclusiva responsabilidad de los autores y no necesariamente reflejan el pensamiento de la Revista Visionario Digital.

El artículo queda en propiedad de la revista y, por tanto, su publicación parcial y/o total en otro medio tiene que ser autorizado por el director de la Revista Visionario Digital.
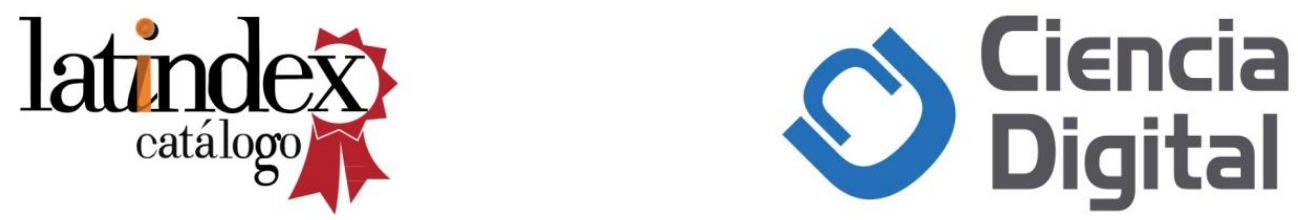